\title{
ON THE RITT ORDER OF A CERTAIN CLASS OF FUNCTIONS
}

\author{
R. PARVATHAM and J. THANGAMANI \\ The Ramanujan Institute \\ University of Madras \\ Madras-600 005, INDIA \\ (Received August 14, 1981)
}

ABSTRACT. The authors introduce the notions of Ritt order and lower order to functions defined by the series $\sum_{1}^{\infty} f_{n}(s) \exp \left(-\lambda_{n} s\right)$ where $\left(\lambda_{n}\right)$ is a D-sequence and $f_{n}(s)$ are entire functions of bounded index.

KEY WOROS AND PHRASES. Ritt order, entire functions of bounded index, Dirichlet series.

1980 MATHEMATICS SUBJECT CLASSIFICATION CODES. 30A16, 30A64.

\section{INTRODUCTION.}

Let us consider an M-dirichletian element:

$$
\{\phi\}: \sum_{n=1}^{\infty} f_{n}(s) \exp \left(-\lambda_{n} s\right), \quad s=\sigma+i \tau, \quad(\sigma, \tau) \varepsilon \frac{2}{\mathbb{R}}
$$

where $\left(\lambda_{n}\right)$ is a D-sequence (a strictly increasing unbounded sequence of positive numbers) and $f_{n}(s)$ are entire functions of bounded index (defined below). Convergence properties of such elements were discussed by J.S.J. Mac Donnell in his doctoral dissertation [1] under the conditions $\lim _{n \rightarrow \infty} \frac{\log n}{\lambda_{n}}=0$ and $\underset{n \rightarrow \infty}{\lim } \frac{m_{n}}{\lambda_{n}}=0$ where $m_{n}$ is the index of $f_{n}$. In this paper, we first study the convergence properties of these elements with less restrictions, namely,

$$
\begin{aligned}
& L=\lim _{n \rightarrow \infty} \sup \frac{\log n}{\lambda_{n}}<\infty \text { and } \\
& B=\lim _{n \rightarrow \infty} \sup \frac{m_{n}}{\lambda_{n}}<\infty
\end{aligned}
$$

As the functions defined by these series are unbounded in the half-plane, it is not possible to define Ritt order directly. However, by making use of functions 
defined by associated intermediate series, we introduce the notions of Ritt order and lower order to these functions.

\section{MAIN RESULTS.}

DEFINITION 2.1. [2 $\mathrm{j}$. An entire function $\mathrm{f}$ is said to be of bounded index if there exists a non-negative integer $\mathrm{N}$ such that

$$
\max _{0 \leq k \leq N}\left\{\frac{\left.\mid \mathrm{f}^{(\mathrm{k})}(\mathrm{s})\right\rfloor}{\mathrm{k} !}\right\} \geq \frac{\left\lfloor\mathrm{f}^{(j)}(\mathrm{s}) \mid\right.}{j !}\left(\mathrm{f}^{(0)}(\mathrm{s})=\mathrm{f}(\mathrm{s})\right)
$$

for $a 11 \mathrm{j}$ and for all s. The least such integer $\mathrm{N}$ is called the index of $\mathrm{f}$.

We require the following lemma which shows that an entire function of bounded index is of exponential type.

LEMNA 2.2. [3], [2]. Let $\mathrm{f}$ be an entire function of bounded index $\mathrm{N}$. Then

$$
|f(s)| \leq\left\{\max _{0 \leq k \leq N} \frac{\left|f^{(k)}(0)\right|}{(N+1)^{k}}\right\} \exp (N+1)|s| .
$$

Let $f_{n}(s)=\sum_{j=0}^{\infty} a_{n j} s^{j}$ be an entire function of bounded index $m_{n}$;

$$
\left.A_{n}=\max \left\{\left|a_{n_{j}}\right| / j=0,1, \ldots, m_{n}\right\}=\max \frac{|f(j)(0)|}{j !}, j=0,1, \ldots, m_{n}\right\}
$$

$\{X\}: \sum_{1}^{\infty} A_{n} \exp \left(-\lambda_{n} s\right)$ the associated dirichletian element whose abscissa of convergence is denoted by $\sigma_{c}^{X} ; k=\lim _{n \rightarrow \infty} \frac{\log A_{n}}{\lambda_{n}}$.

REMARK 2.3. It can be easily seen from Lemma 2.2 that

$$
\begin{aligned}
\left|f_{n}(s)\right| & \leq \max _{0 \leq j \leq m_{n}}\left\{\frac{\left|f_{n}^{(j)}(0)\right|}{j !}\right\} \exp \left(m_{n}+1\right)|s| \\
& =A_{n} \exp \left(m_{n}+1\right)|s| .
\end{aligned}
$$

THEOREM 2.4. If $0 \leq \beta<1$, the region of absolute convergence of (1.1) is the exterior of the hyperbola centre $\left(k\left(1-\beta^{2}\right)^{-1}, 0\right)$ and eccentricity $\beta^{-1}$ contained in the half-plane $\sigma>k$.

PROOF. Using Remark 2.3, we have

$$
\left|\mathrm{f}_{\mathrm{n}}(\mathrm{s}) \exp \left(-\lambda_{\mathrm{n}} \mathrm{s}\right)\right| \leq \mathrm{A}_{\mathrm{n}} \exp \left(\mathrm{m}_{\mathrm{n}}+1\right)|\mathrm{s}| \exp \left(-\lambda_{\mathrm{n}} \sigma\right) .
$$


From the definitions of $k$ and $\beta$ it follows that for $\varepsilon>0$

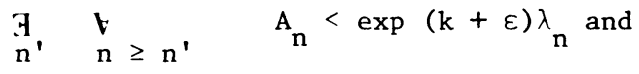

$$
\begin{aligned}
& \mathrm{J}_{\mathrm{n}} \quad \mathrm{n}_{\mathrm{n}} \geq \mathrm{n}^{\prime \prime} \quad \mathrm{m}_{\mathrm{n}}+1<(\beta+\varepsilon) \lambda_{\mathrm{n}} .
\end{aligned}
$$

Hence

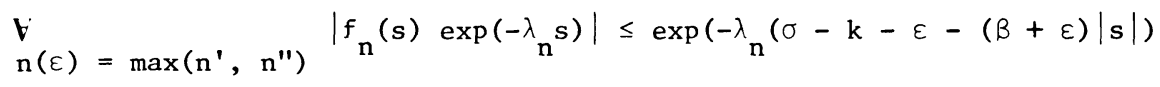

and

$$
\sum_{n(\varepsilon)}^{\infty} f_{n}(s) \exp \left(-\lambda_{n} s\right) \leq \sum_{n(\varepsilon)}^{\infty} \exp \left(-\lambda_{n}(\sigma-k-\varepsilon-(\beta+\varepsilon)|s|) .\right.
$$

The series in the right hand side converges provided

$$
\sigma-k-\beta|s|>0
$$

which is valid only if $\sigma>k$ and $0 \leq \beta<1$.

Thus any point in the region of convergence of (1.1) must satisfy

$$
(\sigma-k)^{2}-\beta^{2}\left(\sigma^{2}+\tau^{2}\right)>0
$$

which reduces to

$$
\left(\sigma-\frac{k}{1-\beta^{2}}\right)^{2}-\frac{\beta^{2} \tau^{2}}{1-\beta^{2}}>\frac{k^{2} \beta^{2}}{\left(1-\beta^{2}\right)^{2}}
$$

from which the theorem follows.

REMARK 2.5. If $\beta=0$ the M-dirichletian element converges in the half-plane $\sigma>k$ (which coincides with the half-plane of convergence of the associated series $\{x\})$ thus giving the result of MacDonnell [1] as a particular case.

Next we proceed to introduce the notions of Ritt order and lower order for functions defined by (1.1). We need the following lemmas.

Let the M-dirichletian element given by (1.1) converge absolutely on $\mathrm{E}_{\mathrm{a}}^{\phi}$ and $\mathrm{D}_{\mathrm{O}}=\{\mathrm{s} \varepsilon \mathrm{C} \mid \sigma=0, \tau \varepsilon \mathbb{R}\}$ denote the imaginary axis.

LEMMA 2.6. Under the conditions $\sigma_{c}^{X}=-\infty$ and $0 \leq \beta<\infty$, we have $E_{a}^{\phi}=\phi$ and $\phi$ is holomorphic on $\$$.

PROOF. Using (2.3) we have

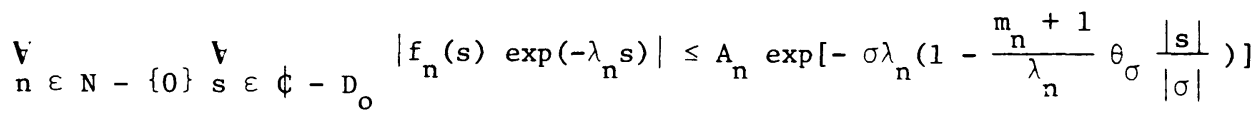

where $\theta_{\sigma}=1$ if $\sigma>0$ and $\theta_{\sigma}=-1$ if $\sigma<0$. 
Since $0 \leq \beta<\infty$ given $\varepsilon>0$

$$
\begin{aligned}
& \mathrm{A}^{\prime}\left(=\mathrm{n}_{\epsilon}\right) \quad \stackrel{\forall}{\mathrm{n} \geq \mathrm{n}^{\prime}} \quad \stackrel{\forall}{\mathrm{s} \varepsilon \phi-D_{0}}\left|\mathrm{f}_{\mathrm{n}}(\mathrm{s}) \exp \left(-\lambda_{\mathrm{n}} \mathrm{s}\right)\right| \\
& \leq A_{n} \exp \left[-\sigma \lambda_{n}\left(1-(\beta+\varepsilon)\left|\frac{s}{\sigma}\right| \theta_{\sigma}\right)\right] \text {. }
\end{aligned}
$$

For any point (on the imaginary axis) $s_{0}=i \tau_{0}$ of $D_{0}$ we extend $\frac{\sigma|s|}{|\sigma|} \theta_{\sigma}$ by its limiting value as $s \rightarrow s_{0}$.

The function $\phi \rightarrow s \rightarrow \sigma\left[1-(\beta+\varepsilon) \frac{|s|}{|\sigma|} \theta_{\sigma}\right]$ is continuous on $\phi$. Let $\mathrm{E}_{\mu}=\left\{\mathbf{s} \varepsilon \phi / \sigma\left(1-(\beta+\varepsilon) \frac{|\mathbf{s}|}{|\sigma|} \theta_{\sigma}\right) \geq \mu\right\}$ indexed by $\mu$ on $\mathbf{R}$. Then $\left\{\phi_{\mathbf{n}},\right\}$ converges uniformly on each $E_{\mu}$ as $\mu>\sigma_{c}^{X}$, where

$$
\left\{\phi_{n^{\prime}}\right\}: \sum_{n=n^{\prime}}^{\infty} f_{n}(s) \exp \left(-\lambda_{n} s\right) .
$$

Let $G$ be any open subset of $\$$. We put

$$
\mu_{G}=\inf \left\{\sigma\left(1-(\beta+\varepsilon) \frac{|s|}{|\sigma|} \theta_{\sigma}\right) \mid s \varepsilon G\right\} .
$$

The number $\mu_{G}$ is fitte $j_{-}$-nd $\left\{\phi_{n},\right\}$ converges absolutely in $G$ if $\sigma_{c}^{X}=-\infty$; further, $\phi_{G}: \quad G \rightarrow s \rightarrow \phi(s)$ is holmorphic on G. Since G is arbitrary on $\phi,\{\sigma\}$ converges absolutely on each point of $\phi$ and $\phi_{G}$ can be continued analytically on the totality of $\phi$. Let $\phi$ denote its analytic continuation. Now we put

$$
\begin{aligned}
& \forall \in \mathbb{R} \\
& \sigma \in M^{\phi}(\sigma, B)=\sup \left\{\left|\phi\left(s^{\prime}\right)\right| / \sigma^{\prime} \geq \sigma \quad s \in B\left(\tau_{1}, \ell\right)\right\}
\end{aligned}
$$

where $B\left(\tau_{1}, \ell\right)=\left\{s \in \phi /\left|\tau-\tau_{1}\right| \leq \ell\right\}$ is the horizontal strip with $\tau=\tau_{1}$ as axis and of width $2 l$. Then

LEMMA 2.7. Under the conditions $\sigma_{c}^{X}=-\infty$ and $0 \leq \beta<1$ we have $\underset{1}{\forall}, \ell) \varepsilon \mathbb{R} \times \mathbb{R}_{0}^{+} M^{\phi}(\sigma, B)$ is bounded on each point $\sigma \in \mathbb{R}$ and $\lim M^{\phi}(\sigma, B)=0$ as $\sigma \rightarrow+\infty$.

PROOF. Let $\left(\tau_{1}, \ell\right)$ be fixed arbitrarily on $\mathbb{R} \times \mathbb{R}_{0}^{+}$. Then given $\varepsilon^{\prime}>0$, ${\stackrel{J}{\sigma} \varepsilon^{\prime}}^{\forall} \quad \quad s \in\left\{\sigma \geq \sigma_{\varepsilon^{\prime}} / s \in B\left(\tau_{1}, \ell\right)\right\} \frac{|s|}{|\sigma|}<1+\varepsilon^{\prime}$ and $\quad$ with $0 \leq \beta<1$. We have 


$$
\begin{aligned}
\underset{\sigma \geq \sigma_{\varepsilon^{\prime}}}{\sigma} \sigma\left(1-(\beta+\varepsilon) \frac{|s|}{|\sigma|} \theta_{\sigma}\right) & >\sigma\left(1-(\beta+\varepsilon)\left(1+\varepsilon^{\prime}\right)\right) \\
& 2 \sigma_{\varepsilon}\left(1-(\beta+\varepsilon)\left(1+\varepsilon^{\prime}\right)\right) ;
\end{aligned}
$$

as a result of $(2,6)$ and $(2.7)$

$$
\begin{aligned}
M^{\phi^{\prime}(\sigma, B)} & <A_{n^{\prime}} \exp \left(-\sigma_{\varepsilon^{\prime}}\left(1-(\beta+\varepsilon)\left(1+\varepsilon^{\prime}\right)\right)\right. \\
& =X_{n^{\prime}}\left(\sigma_{\varepsilon^{\prime}}\left(1-(\beta+\varepsilon)\left(1+\varepsilon^{\prime}\right)\right)\right.
\end{aligned}
$$

and hence

finally,

$$
\begin{aligned}
& \lim M^{\phi_{n}}(\sigma, B)=0 \text { as } \sigma \rightarrow \infty \\
& \lim M^{\phi}(\sigma, B)=0 \text { as } \sigma \rightarrow \infty \text {. }
\end{aligned}
$$

Further, we have

$$
\underset{\sigma}{t} \geq-\sigma_{\varepsilon^{\prime}} \sigma\left(1-(\beta+\varepsilon) \frac{|s|}{|\sigma|} \theta_{\sigma}\right)>\sigma\left(1+(\beta+\varepsilon)\left(1+\varepsilon^{\prime}\right)\right) \text {; }
$$

we put $M\left(\sigma_{\varepsilon^{\prime}}\right)=\operatorname{Max}\left\{\left|\phi_{n^{\prime}}(s)\right| / \sigma \leq \sigma_{\varepsilon^{\prime}} \wedge s \varepsilon B\left(\tau_{1}, l\right)\right.$. As $\phi_{n^{\prime}}$ is holomorphic on the compact set, $\left\{s \in B\left(\tau_{1}, \ell\right) /|\sigma| \leq \sigma_{\varepsilon}\right\}, M\left(\sigma_{\varepsilon}^{\prime}\right)$ is finite. Then we get

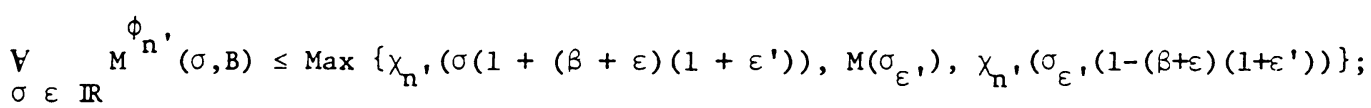

$X_{n}$, is a strictly decreasing function in $\mathbb{R}$ and hence

$$
\begin{aligned}
& \chi_{n^{\prime}}\left(\sigma[1+\beta+\varepsilon)\left(1+\varepsilon^{\prime}\right)\right]>\chi_{n^{\prime}}\left(\sigma\left(1-(\beta+\varepsilon)\left(1+\varepsilon^{\prime}\right)\right) \text { if } \sigma<0\right. \text { and } \\
& \chi_{n^{\prime}}\left(\sigma\left(1+(\beta+\varepsilon)\left(1+\varepsilon^{\prime}\right)\right)<\chi_{n^{\prime}}\left(\sigma\left(1-(\beta+\varepsilon)\left(1+\varepsilon^{\prime}\right)\right) \text { if } \sigma>0,\right.\right.
\end{aligned}
$$

(equality holds for $\sigma=0$ ) with $\chi_{n},(\sigma)=0$ as $\sigma \rightarrow \infty$.

As all M-dirichletian polynomials satisfy the two properties of the lemma, in each horizontal strip $B(\tau, \ell) M^{\phi}(\sigma, B)$ is bounded for each $\sigma \varepsilon \mathbb{R}$ and hence the function $\sigma \rightarrow M^{\phi}(\sigma, B)$ is decreasing on $\mathbb{R}$ with $\lim _{\sigma \rightarrow \infty} M^{\phi}(\sigma, B)=0$.

DEFINITION 2.8. We put

$$
\rho_{B}^{\phi}=\lim _{\sigma \rightarrow-\infty} \sup _{-\infty}\left\{\frac{\log \log ^{+} M^{\phi}(\sigma, B)}{-\sigma}\right\} \text {. }
$$

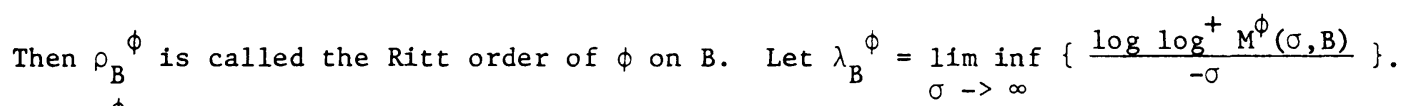
Then $\lambda_{B} \phi$ is called the lower order of $\phi$ on $B$.

THEOREM 2.9. Under the conditions $\sigma_{c} X=-\infty$ and $0 \leq \beta<1$, we have

$$
\underset{1}{\forall}, \ell) \varepsilon \mathbb{R} \times \mathbb{R}_{0}^{+} \rho_{\mathrm{B}}^{\phi} \leq \rho_{\mathrm{R}}^{X} \text { and } \lambda_{\mathrm{B}}^{\phi} \leq \lambda_{\mathrm{R}}^{X}
$$


where $\rho_{R}^{X}$ and $\lambda_{R}^{X}$ are respectively the Ritt order and lower order of $X$ in the whole plane.

PROOF. Proceeding as in Lemma 2.6 for $0 \leq \beta<1$, we have by $(2.6)$

$$
\stackrel{\forall}{\forall} \varepsilon \mathbb{R}_{0}^{+}{\stackrel{\mathrm{n}}{\prime}\left(=\mathrm{n}_{\varepsilon}\right)}_{\mathrm{s} \varepsilon B\left(\tau_{1}, l\right)}^{\forall} \quad\left|\phi_{\mathrm{n}^{\prime}}(\mathrm{s})\right|<x_{\mathrm{n}^{\prime}}\left[\sigma\left(1-(\beta+\varepsilon) \frac{|s|}{|\sigma|} \theta_{\sigma}\right)\right] .
$$

Now denoting by $\phi_{n}$, and $\chi_{n}$, the holomorphic functions on $\phi$ defined by the elements $\left\{\phi_{n},\right\}$ and $\left\{\chi_{n},\right\}$, we have for $\sigma$ negative with $|\sigma|$ sufficiently large:

$$
M^{\phi}{ }^{\prime}(\sigma, B) \leq \chi_{n^{\prime}}\left[\sigma\left(1-(B+\varepsilon) \frac{|s|}{|\sigma|} \theta_{\sigma}\right)\right]
$$

which gives

$$
\underset{\varepsilon^{\prime} \varepsilon \mathbb{R}_{0}^{+}}{\forall} \rho_{\mathrm{B}^{\prime}}^{\boldsymbol{n}^{\prime}} \leq \rho_{\mathrm{R}^{n^{\prime}}}^{\chi}\left[1+(B+\varepsilon) \varepsilon^{\prime}\right]
$$

and hence $\rho_{B^{\prime}}^{\phi} n^{\prime} \leq \rho_{R^{\prime}}^{X}$.

As adding finite number of terms to a holomorphic function defined by a classical

Dirichlet series does not affect its Ritt order [4], we add $\sum_{n=0}^{n^{\prime}-1} A_{n} \exp \left(-\lambda_{n} s\right)$ to $\left\{\chi_{n^{\prime}}\right\}$ and then $\rho_{R^{\prime}}^{X}=\rho_{R^{\prime}}^{X}$.

Now

$$
\forall_{\sigma \varepsilon \mathbb{R}} M^{\phi}(\sigma, B) \leq M^{\phi} n^{\prime}(\sigma, B)+M^{\phi^{\prime}}(\sigma, B)
$$

where $\left\{\phi_{n^{\prime}}^{0}\right\}: \sum_{n=1}^{n-1} f_{n}(s) \exp \left(-\lambda_{n} s\right)$; then

$$
\begin{aligned}
\underset{(\tau, \ell) \varepsilon \mathbb{R} \times \mathbb{R}_{0}^{+}}{\forall} \rho_{B}^{\phi} & \leq \max \left(\rho_{B^{\prime}}^{\phi}, \rho_{B^{\prime}}^{\phi^{\prime}}\right) \\
& =\rho_{B^{\prime}}^{\phi} \text { since } \rho_{B^{\prime}}^{\phi^{\prime}}=0 .
\end{aligned}
$$

Finally we have

$$
\underset{(\tau, \ell) \varepsilon \mathbb{R}^{\forall} \mathbb{R}_{0}^{+}}{\forall} \rho_{\mathrm{B}}^{\phi} \leq \rho_{\mathrm{R}}^{X}
$$

and similarly we can show:

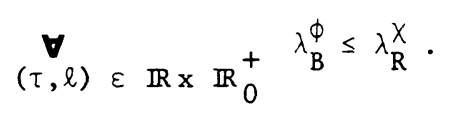

Now we are in a position to define the Ritt order and lower order of $\phi$ in the whole plane $\phi$. 
DEFINITION 2.10. We put

and

$$
\rho_{\mathrm{R}}^{\phi}=\sup \left\{\rho_{\mathrm{B}}^{\phi} /\left(\tau_{1}, \ell\right) \varepsilon \mathbb{R} \times \mathbb{R}_{0}^{+}\right\}
$$

$$
\lambda_{\mathrm{R}}^{\phi}=\sup \left\{\lambda_{\mathrm{B}}^{\phi} /\left(\tau_{1}, \ell\right) \varepsilon \mathbb{R} \times \mathbb{R}_{0}^{+}\right\}
$$

Then $\rho_{R}^{\phi}$ is called the Ritt order of $\phi$ on $\phi$ and $\lambda_{R}^{\phi}$ is called the lower order of $\phi$ on $\phi$.

\section{REFERENCES}

1. MAC DONNELL, J.S.J. Doctoral dissertation, The Catholic University of America, (1957).

2. SHAH, S.M. Entire functions of bounded index, Proc. Amer. Math. Soc. 19, (1968) 1017-1022.

3. HAYMAN, W.K. Differential inequalities and local valency, Pacific J. Math. 44, (1973) 117-137.

4. BLAMBERT, M. Sur la comparison des ordes boreliens et (R) des functions entieres d'une certaene classe, Bull. Sc. Math. 89, (1965) 103-125. 


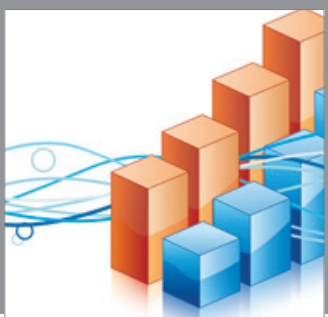

Advances in

Operations Research

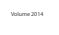

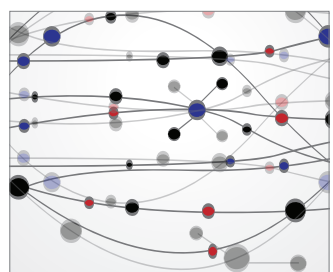

\section{The Scientific} World Journal
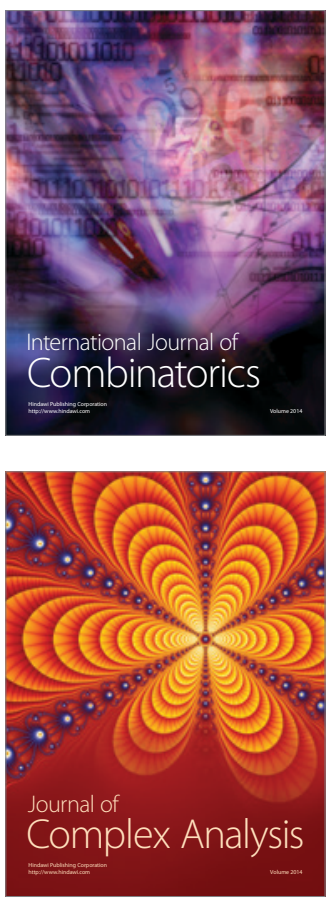

International Journal of

Mathematics and

Mathematical

Sciences
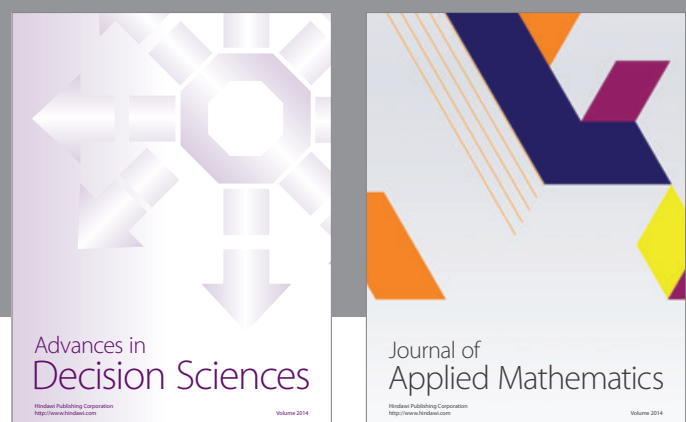

Journal of

Applied Mathematics
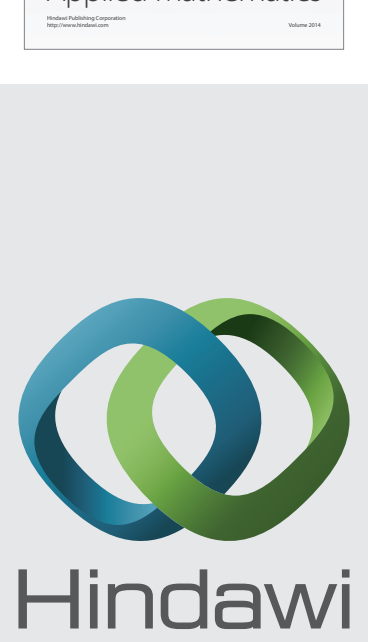

Submit your manuscripts at http://www.hindawi.com
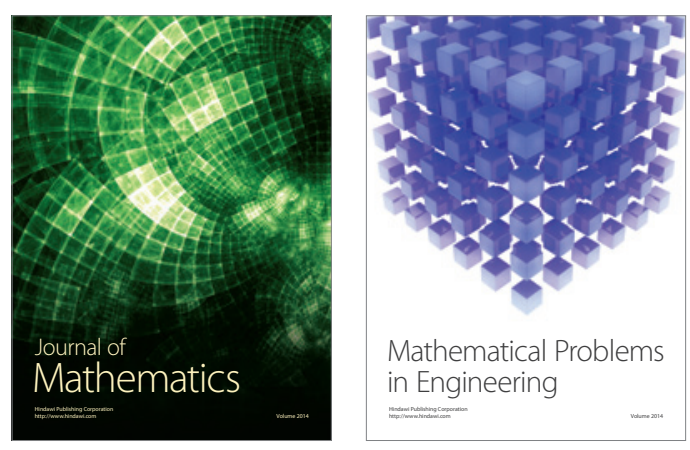

Mathematical Problems in Engineering
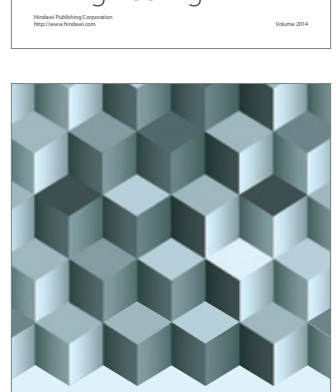

Journal of

Function Spaces
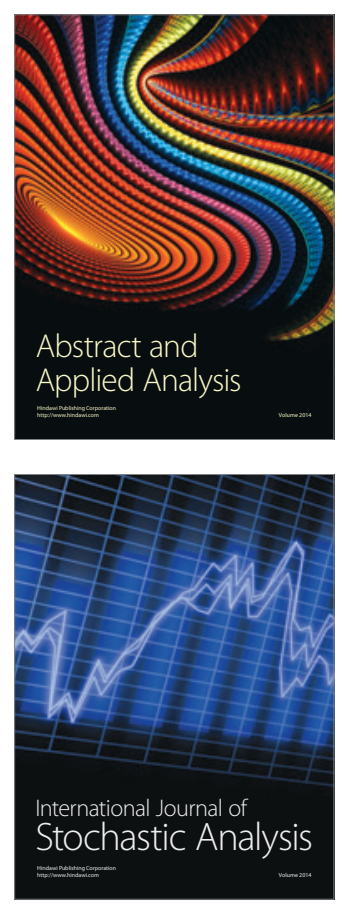

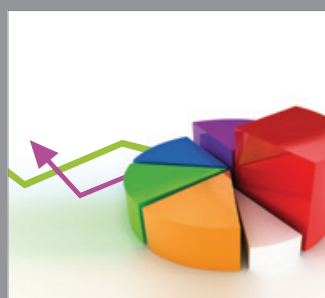

ournal of

Probability and Statistics

Promensencen
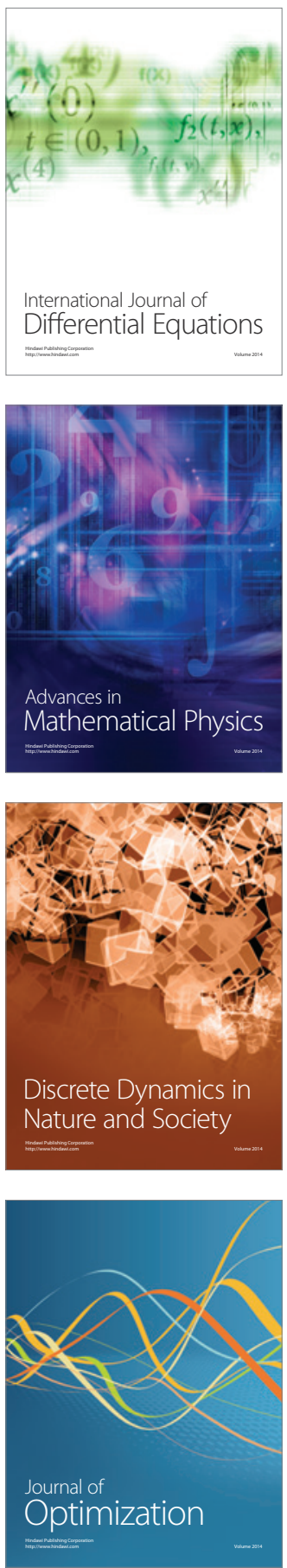